\title{
A characterization of generalized quaternion 2-groups
}

\author{
Marius Tărnăuceanu \\ Faculty of Mathematics, "Al.I. Cuza" University, Iaşi, Romania
}

\begin{abstract}
The goal of this note is to give a characterization of generalized quaternion 2-groups by using their posets of cyclic subgroups.
\end{abstract}

Key words: generalized quaternion 2-groups, cyclic subgroups, posets, breaking points.

2000 MSC: Primary 20E07; Secondary 20E15, 20F22.

\section{Résumé}

Une caractérisation de groupes de quaternions généralisés. Le but de cette note est de donner une caractérisation de 2-groupes de quaternions généralisés en utilisant leur ensembles partiellement ordonnés de sous-groupes cycliques.

\section{Introduction}

Let $G$ be a finite group and $L(G)$ be the subgroup lattice of $G$. The starting point for our discussion in given by the paper [1, where the proper nontrivial subgroups $H$ of $G$ with the property that

$$
\text { for every } X \in L(G) \text { we have } X \leq H \text { or } H \leq X
$$

have been studied. Such a subgroup is called a breaking point for the lattice $L(G)$. Clearly, if $L(G)$ is a chain (i.e. $G$ is a cyclic $p$-group), then all proper nontrivial subgroups $H$ of $G$ are breaking points. On the other hand, we

Email address: tarnauc@uaic.ro (Marius Tărnăuceanu). 
remark that the above concept can naturally be extended to other remarkable posets of subgroups of $G$ (and also to arbitrary posets). One of them is the poset of cyclic subgroups of $G$, denoted usually by $C(G)$. The study of the existence and of the uniqueness of breaking points in $C(G)$ constitutes the purpose of this paper.

Most of our notation is standard and will usually not be repeated here. Elementary concepts and results on group theory can be found in [2] and [4]. For subgroup lattice notions we refer the reader to [3] and [5].

We mention that by a generalized quaternion 2-group we mean a group of order $2^{n}$ for some natural number $n \geq 3$, defined by the presentation

$$
Q_{2^{n}}=\left\langle a, b \mid a^{2^{n-2}}=b^{2}, a^{2^{n-1}}=1, b^{-1} a b=a^{-1}\right\rangle .
$$

We also recall that these groups are the unique finite noncyclic $p$-groups all of whose abelian subgroups are cyclic, or equivalently the unique finite noncyclic $p$-groups possessing exactly one subgroup of order $p$ (see (4.4) of [4], II). Obviously, this result shows that the subgroup of order 2 of $Q_{2^{n}}$, namely $\left\langle a^{2^{n-2}}\right\rangle$, is the unique breaking point of $C\left(Q_{2^{n}}\right)$.

Our main theorem proves that generalized quaternion 2-groups exhaust all finite noncyclic groups whose posets of cyclic subgroups have breaking points.

Theorem 1.1. Let $G$ be a finite group. Then $C(G)$ possesses breaking points if and only if $G$ is either a cyclic p-group of order at least $p^{2}$ or a generalized quaternion 2-group.

\section{The proof of Theorem 1.1}

We observe first that the above theorem can be easily proved in the particular case of $p$-groups.

Lemma 2.1. Let $G$ be a finite p-group. Then $C(G)$ possesses breaking points if and only if $G$ is either a cyclic p-group of order at least $p^{2}$ or a generalized quaternion 2-group.

Proof. Suppose that $G$ is not cyclic and let $H$ be a breaking point of $C(G)$. Then all minimal subgroups $M_{1}, M_{2}, \ldots, M_{k}$ of $G$ are contained in $H$. If $k \geq 2$, then we infer that $H$ is not cyclic, a contradiction. So, we have $k=1$, that is $G$ has a unique subgroup of order $p$. This implies that $G$ is a generalized quaternion 2-group, according to the result mentioned in Section 1.

The converse implication is obvious, completing the proof. 
We are now able to give a proof of Theorem 1.1 .

Proof. Suppose that the poset $C(G)$ of cyclic subgroups of a finite group $G$ possesses a breaking point, say $H$.

In the following we shall focus on proving that $G$ must necessarily be a $p$ group. By the way of contradiction, assume that the order of $G$ has at least two distinct prime divisors. Clearly, the same thing can be also said about the order of $H$. Let $p \in \pi(G)$ and $K$ be a cyclic $p$-subgroup of $G$. Since $H$ is not a $p$-subgroup, we infer that $K \subseteq H$. In other words, $H$ contains any cyclic $p$-subgroup of $G$ and consequently any $p$-element of $G$. This implies that all Sylow $p$-subgroups of $G$ are contained in $H$. Then $H=G$, a contradiction.

Hence $G$ is a $p$-group, for some prime $p$, and now the conclusion follows from Lemma 2.1.

By the above results we also infer that, given a finite group $G$, the poset $C(G)$ possesses a unique breaking point if and only if $G$ is either a cyclic $p$-group of order $p^{2}$ or a generalized quaternion 2-group. In other words, the following corollary holds.

Corollary 2.2. The generalized quaternion 2-groups are the unique finite noncyclic groups whose posets of cyclic subgroups have exactly one breaking point.

Finally, we indicate a natural generalization of our study, suggested by the reviewers of the paper. Let $G$ be a finite group and denote by

$$
\bar{C}(G)=\{[H] \mid H \in C(G)\}
$$

the set of conjugacy classes of cyclic subgroups of $G$. Mention that $\bar{C}(G)$ is also a poset under the ordering relation

$$
\left[H_{1}\right] \leq\left[H_{2}\right] \text { if and only if } H_{1} \subseteq H_{2}^{g} \text {, for some } g \in G \text {. }
$$

Take a breaking point $[H]$ of $\bar{C}(G)$. Then $H \in C(G)$ satisfies the following condition: for any cyclic subgroup $C$ of $G$, some conjugate of $C$ in $G$ contains or is contained in $H$. Clearly, this is weaker than the condition that $H$ be a breaking point of $C(G)$. We remark that for a finite $p$-group $G$ it is sufficient to guarantee the uniqueness of a subgroup of order $p$ in $G$. In other words, Lemma 2.1 also holds if we replace $C(G)$ with $\bar{C}(G)$. In the general case, that is for arbitrary finite groups $G$, the problem of characterizing the existence and the uniqueness of breaking points of $\bar{C}(G)$ remains still open. 


\section{Conclusions and further research}

All previous results show that the concept of breaking point in some posets of subgroups of a (finite) group $G$ can constitute an important aspect of subgroup lattice theory. Clearly, its study started in [1] for lattices of subgroups and continued in the present paper for posets of cyclic subgroups can successfully be extended to other significant lattices/posets associated to $G$ (as the lattice of normal/subnormal/characteristic/solitary subgroups of $G$ or the poset of centralizers/conjugacy classes of elements (subgroups) of $G$ ). Studying the breaking points of arbitrary posets (not necessarily connected with a group $G$ ) seems to be also very interesting. These will surely constitute the subject of some further research.

Acknowledgements. The author is grateful to the reviewers for their remarks which improve the previous version of the paper.

\section{References}

[1] Gr.G. Călugăreanu, M. Deaconescu, Breaking points in subgroup lattices, Proceedings of Groups St. Andrews 2001 in Oxford (Cambridge University Press 2003), vol. 1, 59-62.

[2] B. Huppert, Endliche Gruppen, I, Springer Verlag, Berlin-Heidelberg-New York, 1967.

[3] R. Schmidt, Subgroup lattices of groups, de Gruyter Expositions in Mathematics 14, de Gruyter, Berlin, 1994.

[4] M. Suzuki, Group theory, I, II, Springer Verlag, Berlin, 1982, 1986.

[5] M. Tărnăuceanu, Groups determined by posets of subgroups, Ed. Matrix Rom, Bucureşti, 2006. 